\title{
Implementation of Therapeutic Drug Monitoring and Pharmacogenetic Tests in Psychiatry: How About ABCB1?
}

\section{Pierre Baumann*}

\author{
Department of Psychiatry (DP-CHUV), Université de Lausanne Site de Cery, CH-1008 Prilly-Lausanne, Switzerland
}

Pharmacotherapy in psychiatry is characterised by a clinical response of patients frequently delayed by several days or even weeks. Moreover, there are no firm biological parameters which can be monitored to get an objective picture of the clinical outcome in patients suffering from schizophrenia or an affective disorder, except for some adverse effects. Therapeutic drug effects are often subtle, changes in psychopathology have to be measured using adequate rating scales. Such scales are also used for the qualitative and quantitative analysis of adverse effects such as sedation, inner tension, anxiety, suicidal ideas, extrapyramidal symptoms. On the other hand, laboratory exams have to be included to monitor possible adverse effects at e.g. the haematological level $[1,2]$. Besides, patients often lack adherence to the treatment. This situation but also environmental, personal and genetic factors are responsible for a high interindividual variability of the metabolism and pharmacokinetics in the subjects. This variability has consequences on the pharmacodynamics of the therapeutic agents. Therefore, therapeutic drug monitoring and pharmacogenetics tests have been introduced as valid instruments to optimise treatment [3].

Most psychotropic drugs such as antidepressants and antipsychotics are metabolised by genetically polymorphic forms of cytochrome P-450 (CYP) and some of them are also substrates and/or inhibitors/inducers of the transporter protein ABCB1 (P-glycoprotein $(\mathrm{PgP})$. Recently, several groups of authors have raised the question whether time has come for implementation of pharmacogenomics in psychiatry in order to promote personalized therapy [4]. While the general strategy of TDM includes consideration of the role of CYP in drug-drug interactions and in the pharmacogenetic factors responsible for the variability of drug plasma concentrations, this seems to be far less the case with $\operatorname{PgP}[3]$.

PgP belongs to the family of the ATP-binding cassette (ABC) transporters and acts as an efflux transporter in that it limits the access of drugs and other xenobiotics to the organism at different levels, e.g. in the intestine, kidney and liver, but also at the blood-brain barrier [5]. This protein is characterised by multiple membrane-spanning domains that determine a pore and it contains intracellular nucleotidebinding domains for ATP-dependent translocation of substrates across the cell membrane [6]. Psychotropic drugs such as e.g. citalopram but not mirtazapine is PgP substrates. PgP is encoded by the polymorphic ABCB1 gene. Thousands of SNPs have been described, including several hundred in the coding region. This gave rise to the hypothesis that genetic variants of $\operatorname{PgP}$ differ in their ability to influence the transport of drugs and substrates. In one of the first studies, the authors observed that in depressive patients treated with PgP substrates (incl. amitriptyline, citalopram, venlafaxine and others), there was an association between several SNPs of ABCB1 and their clinical outcome [7]. However, there are conflicting results about the effect of the genetic variability of $\mathrm{ABCB} 1$ on the clinical outcome of patients treated with antidepressants. Some authors [8] therefore recommend ABCB1 genotyping, but others conclude that there is insufficient evidence to propose $\mathrm{ABCB} 1$ genotyping in clinical routine conditions [9-11].

Therefore, it is valuable that there exist independent organisations, such as the Clinical Pharmacogenetics Implementation Consortium (CIPC) (https://cpicpgx.org) and the Pharmacogenomics Knowledgebase (PharmGKB) (https://www.pharmgkb.org/) hosted by the University of Stanford who analyse the literature in this field [1214]. They publish recommendations for the selection of therapeutic drugs and dose adaptations based on the results of pharmacogenetics tests, on information about the relationship between genes and gene variants, and the clinical efficacy and tolerance on the result of the pharmacological treatment. The recommendations are developed for individual drug - gene pairs. Therefore, PharmGKB has defined several levels of evidence (Levels $1 \mathrm{~A}-4$ ) for these relationships (https://www.pharmgkb.org/page/clinAnnLevels). Depending on the evidence for a particular drug - gene pair, a clinical annotation is then produced, and they vary between: "Testing required", "Testing recommended", "Testing actionable", "Testing informative" (lowest recommendation) (https://www.pharmgkb.org/view/drug-labels. do). It is then remarkable that despite drug - gene pairs such as amitriptyline - CYP2D6 (Level 1A) and citalopram - CYP2C19 (1A) reach highest levels, this confers them a clinical annotation by the FDA of (only) "Test actionable". On the other hand, drug - gene pairs of antidepressants such as amitriptyline, citalopram, reach only an evidence level 3 for $\mathrm{ABCB} 1$, and they do not have any FDA annotation. It has to be mentioned that among neuropsychiatric drugs, there is almost only the drug - gene pair carbamazepine - HLA-B*15:02 and HLA-A*31:01 (in the domain of pharmacodynamics) which reaches the highest annotation, namely "Testing required". In summary, this situation does not yield convincing arguments for ABCB1 genotyping in the everyday clinical practice, in contrast to CYP genotyping for well-defined drug - gene pairs.

The question then arises whether present knowledge allows interpretation of the role of $\mathrm{PgP}$ in pharmacokinetic drug - drug interactions. This has been examined very recently in a comprehensive review paper [15]. e.g., drugs which are either inhibitors and/or inducers of PgP could influence the transport of other drugs, substrates of $\mathrm{PgP}$, in the human brain at the blood-brain-barrier. The authors found very few CNS drugs which modulate clearly PgP activity and which interact with other drugs at a level of clinical relevance. This applies only for the PgP inducers carbamazepine and phenytoin, and for the PgP inhibitors fluvoxamine and paroxetine. Others may

*Corresponding author: Pierre Baumann, Professor, Department of Psychiatry (DP-CHUV), Université de Lausanne Site de Cery, CH-1008 Prilly-Lausanne, Switzerland, Tel: 0383935005 E-mail: pierre.baumann@chuv.ch

Received January 23, 2018; Accepted February 13, 2018; Published February 23, 2018

Citation: Baumann P (2018) Implementation of Therapeutic Drug Monitoring and Pharmacogenetic Tests in Psychiatry: How About ABCB1?. J Bioequiv Availab 10: 22-23. doi: 10.4172/0975-0851.1000371

Copyright: @ 2018 Baumann P. This is an open-access article distributed under the terms of the Creative Commons Attribution License, which permits unrestricted use, distribution, and reproduction in any medium, provided the original author and source are credited. 
Citation: Baumann P (2018) Implementation of Therapeutic Drug Monitoring and Pharmacogenetic Tests in Psychiatry: How About ABCB1?. J Bioequiv Availab 10: 22-23. doi: 10.4172/0975-0851.1000371

modulate $\mathrm{PgP}$ as inhibitors or inducers, but the clinical relevance (in vivo) is uncertain. Their list of compounds which do not have or demonstrate uncertain interaction with $\mathrm{PgP}$ is also of high interest. It includes drugs such as amitriptyline and citalopram. While vortioxetine and sertraline are recognized to modulate PgP activity in vitro, in vivo studies suggest that it has no clinical relevance. The examples shown above also illustrate the fact that many drugs do not only interact with Pgp, but that this activity frequently overlaps with their effect on CYP. Carbamazepine is also an inducer of CYP3A4, fluvoxamine is a potent inhibitor of CYP2C19, CYP1A2 and other CYP forms. The authors conclude that interpretation of drug - drug interactions in this context may be complex [15]. A phenotyping probe would represent a precious instrument to determine the degree of interaction of a comedicated drug on PgP activity, but one of the most cited phenotyping probe, digoxin, does not appear to be convenient as it does not fulfil three essential criteria: specificity, sensitivity and feasibility [16]. On the other hand, despite digoxin is also an OATP2 substrate, its use as a test probe for $\mathrm{PgP}$ can yield interesting results, in that e.g. sertraline causes little, clinically irrelevant increases of digoxin plasma concentrations in comparison to those measured in the absence of the antidepressant drug [17]. Unfortunately, no other psychotropic drugs were tested in these conditions. Therefore, further studies are needed to examine the role of PgP in the pharmacokinetics of psychotropic drugs, especially with regard to its pharmacogenomics and its role in drug-drug interactions. As a consequence, physicians should be aware about the role of PgP in potential drug-drug interactions, but other factors have therefore also to be considered.

\section{References}

1. Stahl SM, Morrissette DA, Citrome L, Saklad SR, Cummings MA, et al. (2013) "Meta-guidelines" for the management of patients with schizophrenia. CNS Spectr 18: 150-162.

2. Bauer M, Pfennig A, Severus E, Whybrow PC, Angst J, et al. (2013) World Federation of Societies of Biological Psychiatry (WFSBP) guidelines for biological treatment of unipolar depressive disorders, part 1: update 2013 on the acute and continuation treatment of unipolar depressive disorders. World $\mathrm{J}$ Biol Psychiatry 14: 334-385.

3. Hiemke C, Bergemann N, Clement HW, Conca A, Deckert J, et al (2018) Consensus Guidelines for Therapeutic Drug Monitoring in Neuropsychopharmacology: Update 2017. Pharmacopsychiatry 51: 9-62.
4. Amare AT, Schubert KO, Baune BT (2017) Pharmacogenomics in the treatment of mood disorders: Strategies and Opportunities for personalized psychiatry. EPMA J 8: 211-227.

5. Bruhn O, Cascorbi I (2014) Polymorphisms of the drug transporters ABCB1 $A B C G 2, A B C C 2$ and $A B C C 3$ and their impact on drug bioavailability and clinical relevance. Expert Opin Drug Metab Toxicol 10: 1337-1354.

6. Hodges LM, Markova SM, Chinn LW, Gow JM, Kroetz DL, et al. (2011) Very important pharmacogene summary: ABCB1 (MDR1, P-glycoprotein) Pharmacogenet Genomics 21: 152-161.

7. Uhr M, Tontsch A, Namendorf C, Ripke S, Lucae S, et al. (2008) Polymorphisms in the drug transporter gene ABCB1 predict antidepressant treatment response in depression. Neuron 57: 203-209.

8. Breitenstein B, Bruckl TM, Ising M, Muller-Myhsok B, Holsboer F, et al. (2015) $A B C B 1$ gene variants and antidepressant treatment outcome: A meta-analysis. Am J Med Genet B Neuropsychiatr Genet 168B: 274-283.

9. Bruckl TM, Uhr M (2016) ABCB1 genotyping in the treatment of depression. Pharmacogenomics 17: 2039-2069.

10. Bschor T, Baethge C, Hiemke C, Muller-Oerlinghausen B (2017) Genetic tests for controlling treatment with antidepressants. Nervenarzt 88: 495-499.

11. Wolking S, Schaeffeler E, Lerche H, Schwab M, Nies AT (2015) Impact of Genetic Polymorphisms of ABCB1 (MDR1, P-Glycoprotein) on Drug Disposition and Potential Clinical Implications: Update of the Literature. Clinical Pharmacokinetics 54: 709-735.

12. Whirl-Carrillo M, Sangkuhl K, Gong L, Klein TE (2016) Novel DiseaseDrug Database Demonstrating Applicability for Pharmacogenomic-Based Prescribing. Clin Pharmacol Ther 100: 600-602.

13. Whirl-Carrillo M, McDonagh EM, Hebert JM, Gong L, Sangkuhl K, et al. (2012) Pharmacogenomics knowledge for personalized medicine. Clin Pharmacol Ther 92: 414-417.

14. McDonagh EM, Whirl-Carrillo M, Garten Y, Altman RB, Klein TE (2011) From pharmacogenomic knowledge acquisition to clinical applications: the PharmGKB as a clinical pharmacogenomic biomarker resource. Biomark Med 5:795-806.

15. Lund M, Petersen TS, Dalhoff KP (2017) Clinical Implications of P-Glycoprotein Modulation in Drug-Drug Interactions. Drugs 77: 859-883.

16. Ma JD, Tsunoda SM, Bertino JS, Trivedi M, Beale KK, et al. (2010) Evaluation of in vivo P-glycoprotein phenotyping probes: a need for validation. Clin Pharmacokinet 49: 223-237.

17. Fenner KS, Troutman MD, Kempshall S, Cook JA, Ware JA, et al. (2009) Drugdrug interactions mediated through $\mathrm{P}$-glycoprotein: clinical relevance and in vitro-in vivo correlation using digoxin as a probe drug. Clin Pharmacol Ther 85: 173-181. 